\title{
The Nitric Oxide-cGMP Pathway Controls the Directional Polarity of Growth Cone Guidance via Modulating Cytosolic $\mathrm{Ca}^{2+}$ Signals
}

\author{
Takuro Tojima, ${ }^{\star}$ Rurika Itofusa, ${ }^{\star}$ and Hiroyuki Kamiguchi \\ Laboratory for Neuronal Growth Mechanisms, RIKEN Brain Science Institute, Wako, Saitama 351-0198, Japan
}

\begin{abstract}
Asymmetric $\mathrm{Ca}^{2+}$ signals across the growth cone mediate attractive or repulsive axon guidance depending on the occurrence of $\mathrm{Ca}^{2+}-$ induced $\mathrm{Ca}^{2+}$ release (CICR) through ryanodine receptors (RyRs). Although the neuronal isoform of nitric oxide (NO) synthase (nNOS) is highly expressed in developing dorsal root ganglion (DRG) neurons, the role of NO in axon guidance remains essentially unknown. Here we report that the NO- cGMP pathway negatively regulates CICR to control the directional polarity of DRG axon guidance. Intracellular levels of NO and cGMP depend on extracellular substrates: laminin activates the NO-cGMP pathway, whereas the adhesion molecule L1 does not. The activity of NO and cGMP determines the turning direction of growth cones with respect to asymmetric $\mathrm{Ca}^{2+}$ signals that are produced by photolysing caged $\mathrm{Ca}^{2+}$. The $\mathrm{Ca}^{2+}$ signals cause growth cone repulsion on a laminin substrate, which is converted to attraction by pharmacological blockade of the NO-cGMP pathway or genetic deletion of nNOS. Conversely, $\mathrm{Ca}^{2+}$-induced growth cone attraction on an L1 substrate is converted to repulsion by increasing NO levels. Such NO-mediated switching of turning direction involves the regulation of CICR through RyRs. Furthermore, growth cone repulsion induced by an extracellular gradient of a physiological cue, neurotrophin-4, is dependent on $\mathrm{Ca}^{2+}$ signals and converted to attraction by inhibiting the NO-cGMP pathway. These results suggest that, on contact with different adhesive environments, growth cones can change their turning responses to axon guidance cues by modulating CICR via endogenous NO and cGMP.
\end{abstract}

\section{Introduction}

Nitric oxide (NO), a gaseous messenger synthesized by NO synthases (NOS), is implicated in various processes of neural development, including neuronal migration (Haase and Bicker, 2003) and axon retraction (Trimm and Rehder, 2004; Stroissnigg et al., 2007). NO increases intracellular levels of cGMP by activating soluble guanylyl cyclase (sGC) (Jaffrey and Snyder, 1995; Calabrese et al., 2007). Subsequently, cGMP activates protein kinase G (PKG) that phosphorylates many target proteins, including ryanodine receptors (RyRs) (Suko et al., 1993). Dorsal root ganglion (DRG) neurons express neuronal NOS (nNOS) and PKG in early developmental stages, followed by drastic downregulation of nNOS with age (Ward et al., 1994; Qian et al., 1996; Thippeswamy et al., 2005), implying a role of the nNOS-NOcGMP signaling pathway in nascent DRG neurons. Indeed, it has

\footnotetext{
Received Jan. 7, 2009; revised April 16, 2009; accepted May 19, 2009.

This work was partially supported by Grant-in-Aid for Young Scientists (B) 18700332 (T.T.) and Grant-in-Aid for Scientific Research (C) 19500335 (H.K.) from the Japan Society for the Promotion of Science. T.T. was supported by the RIKEN Special Postdoctoral Researchers Program. We are grateful to A. Guy and H. Akiyama for critical reading of this manuscript. We thank V. Lemmon for providing L1-Fc construct, F. Wada for anti-RyRs antibody, and the Research Resources Center of the RIKEN Brain Science Institute for experimental instruments.

*T.T. and R.I. contributed equally to this work.

Correspondence should be addressed to either Hiroyuki Kamiguchi or Takuro Tojima, Laboratory for Neuronal Growth Mechanisms, RIKEN Brain Science Institute, 2-1 Hirosawa, Wako, Saitama 351-0198, Japan, E-mail: kamiguchi@brain.riken.jp or tojima@brain.riken.jp.

DOI:10.1523/JNEUROSCI.0087-09.2009

Copyright $\odot 2009$ Society for Neuroscience $\quad$ 0270-6474/09/297886-12\$15.00/0
}

been shown that genetic deletion of PKG causes guidance defects of DRG axons in the spinal cord (Schmidt et al., 2002).

The growth cone, a motile ending of an elongating axon, is guided along the correct path by various cues such as netrin-1, semaphorin 3A (Sema3A), and neurotrophins (Tessier-Lavigne and Goodman, 1996; Song and Poo, 1999). An extracellular gradient of these guidance cues increases cytosolic $\mathrm{Ca}^{2+}$, with $\mathrm{Ca}^{2+}$ concentrations highest in the area of growth cone closest to the source of the cues (Henley and Poo, 2004; Henley et al., 2004; Nishiyama et al., 2008). These asymmetric $\mathrm{Ca}^{2+}$ elevations across the growth cone are sufficient to trigger turning to the side with higher $\mathrm{Ca}^{2+}$ (attraction) as well as to the side with lower $\mathrm{Ca}^{2+}$ (repulsion) (Zheng, 2000). It is well known that cAMP modulates the asymmetric $\mathrm{Ca}^{2+}$ signals to determine the turning direction (Nishiyama et al., 2003; Henley et al., 2004). Our previous studies showed that high cAMP activates RyRs and thereby facilitates $\mathrm{Ca}^{2+}$-induced $\mathrm{Ca}^{2+}$ release (CICR) from the endoplasmic reticulum, whereas low cAMP suppresses CICR through RyRs (Ooashi et al., 2005). We also demonstrated that asymmetric $\mathrm{Ca}^{2+}$ signals with and without CICR trigger growth cone attraction and repulsion, respectively. The cAMP levels in growth cones can be regulated by electrical activities (Ming et al., 2001) and environmental factors such as neurotrophins (Gao et al., 2003) and cell adhesion molecules (CAMs) (Höpker et al., 1999; Ooashi et al., 2005). Although less is known about the role of cGMP in axon guidance, cGMP counteracts cAMP and converts growth cone attraction by netrin-1 to repulsion (Nishiyama et al., 2003). Nishiyama et al. (2003) also proposed that cGMP inacti- 
vates L-type voltage-dependent $\mathrm{Ca}^{2+}$ channels and RyRs, although the involvement of RyRs in cGMP-mediated regulation of axon guidance has not been tested experimentally. Furthermore, it is essentially unknown how cGMP levels in growth cones can be controlled by environmental factors.

In the present study, we show that CAMs regulate intracellular levels of NO and cGMP and that the nNOS-NO-cGMP-PKG pathway switches the directional polarity of growth cone turning by regulating CICR through RyRs. We propose that endogenous $\mathrm{NO}$, synthesized by nNOS, links CAM-associated signals to the turning behaviors of growth cones.

\section{Materials and Methods}

Animals. Fertilized chicken eggs (Gallus gallus) were obtained from a local supplier and incubated at $38^{\circ} \mathrm{C}$. Mice lacking nNOS (B6, 129SNos1tm1Plh) were obtained from The Jackson Laboratory and maintained at the RIKEN Brain Science Institute Animal Care Facility.

Cell culture. DRG neurons from embryonic day 9 chicks or postnatal day 0 mice were dissociated as described previously (Ooashi et al., 2005) and plated on a glass-based dish coated with laminin $(\sim 10 \mu \mathrm{g} / \mathrm{ml}$; Invitrogen) or L1-Fc chimeric protein that consists of the ectodomain of the adhesion molecule L1 and the Fc region of human IgG (Kamiguchi and Yoshihara, 2001). The cultures were maintained in Leibovitz's L-15 medium (Invitrogen) supplemented with $\mathrm{N}-2$ (Invitrogen), $20 \mathrm{ng} / \mathrm{ml}$ nerve growth factor (NGF) (Promega), and $750 \mu \mathrm{g} / \mathrm{ml}$ bovine serum albumin (Invitrogen), in a humidified atmosphere of $100 \%$ air at $37^{\circ} \mathrm{C}$.

Intracellular NO measurement. Intracellular NO was measured using the fluorescent NO indicator diaminofluorescein-FM (DAF-FM) (Kojima et al., 1999). Cultured DRG neurons were loaded with 3 or $5 \mu \mathrm{M}$ DAF-FM diacetate (Daiichi), a membrane-permeable analog of DAF$\mathrm{FM}$, for $30 \mathrm{~min}$ at $37^{\circ} \mathrm{C}$, followed by a wash. After additional incubation for 30-60 min, fluorescence images of the neurons were acquired using a $100 \times$ objective (UPLSAPO, oil, numerical aperture 1.40; Olympus) on an inverted microscope (IX71; Olympus) equipped with a CCD camera (ORCA-ER; Hamamatsu Photonics). In some experiments, DAF-FM loading and postincubation were performed in the presence of $50 \mu \mathrm{M}$ 2-(4-carboxyphenyl)-4,4,5,5-tetramethylimidazoline-1-oxyl-3-oxide (PTIO) (Dojindo) or $100 \mu \mathrm{M}$ 1-hydroxy-2-oxo-3,3-bis(2-aminoethyl)1-triazene (NOC18) (Dojindo). PTIO was added $30 \mathrm{~min}$ before the onset of DAF-FM loading to scavenge preexisting NO in the cultures. For quantitative analyses, the background fluorescence was subtracted from the acquired fluorescence images, and the pixel intensities were then averaged within a growth cone or a cell body using AquaCosmos version 2.6 software (Hamamatsu Photonics).

Enzyme immunoassay. For cGMP measurements, chick DRG neurons were cultured for $3 \mathrm{~h}$ on plastic culture plates (12 well, $22 \mathrm{~mm}$ diameter) coated with either laminin or L1-Fc at an initial density of $1 \times 10^{6}$ cells per well. To block the degradation of cyclic nucleotides, the cultures were treated with the phosphodiesterase inhibitor 3-isobutyl-1methylxanthine (IBMX) ( $1 \mathrm{~mm}$; Sigma) for $5 \mathrm{~min}$. During this $5 \mathrm{~min}$ incubation with IBMX, some cultures were cotreated with either $50 \mu \mathrm{M}$ PTIO or $100 \mu \mathrm{M}$ NOC18. Intracellular cGMP concentrations were then measured using a cGMP enzyme immunoassay kit (GE Healthcare) according to the protocol of the manufacturer (acetylation enzyme immunoassay procedure for intracellular cGMP measurement).

For cAMP measurements, neurons were cultured at an initial density of $3-5 \times 10^{5}$ cells per well. Some cultures were treated with PTIO or NOC18 for $5 \mathrm{~min}$. Intracellular cAMP concentrations were measured using a cAMP enzyme immunoassay kit (GE Healthcare) according to the protocol of the manufacturer (non-acetylation enzyme immunoassay procedure for intracellular cAMP measurement).

Growth cone turning assay. Growth cone turning was triggered by focal laser-induced photolysis (FLIP) of the caged $\mathrm{Ca}^{2+}$ compound $o$-nitrophenyl EGTA (NP-EGTA) (Invitrogen) as described previously (Ooashi et al., 2005; Tojima et al., 2007). Spatial restriction of FLIP, as assessed by irradiating caged fluorescein maleimide (Dojindo), was $\sim 1$ $\mu \mathrm{m}$ in diameter in the $x-y$ plane. Growth cone turning was triggered by a microscopic gradient of neurotrophin-4 (NT-4) $(50 \mu \mathrm{g} / \mathrm{ml}$ in pipette;
PeproTech) as described previously (Song et al., 1998; Ming et al., 2001; Henley et al., 2004). In some experiments, $1 \mu \mathrm{M}$ of an AM ester derivative of BAPTA-AM (Invitrogen) was loaded into neurons at least $30 \mathrm{~min}$ before the turning assay experiments, as described previously (Ooashi et al., 2005). The following reagents were applied to some cultures at least 30 min before the turning assays: $100 \mathrm{nM} \mathrm{N} N^{\mathrm{G}}$-monomethyl-L-arginine (L-NMMA) (Tocris Cookson), $50 \mu \mathrm{M}$ PTIO, $100 \mu \mathrm{M}$ 8-bromo-cGMP (8-Br-cGMP) (Calbiochem), $100 \mathrm{~nm} 1 \mathrm{H}$-[1,2,4] oxadiazolo[4,3-a] quinoxalin-1-one (ODQ) (Tocris Cookson), $100 \mu \mathrm{M}$ ryanodine (Latoxan), $10 \mathrm{~nm}(9 S, 10 R, 12 R)-2,3,9,10,11,12$-hexahydro-10-methoxy-2,9dimethyl-1-oxo-9,12-epoxy-1H-diindolo[1,2,3-fg:3', 2' $1^{\prime}{ }^{\prime}$-kl]pyrrolo[3,4i] [1,6] benzodiazocine-10-carboxylic acid methyl ester (KT5823) (Calbiochem), $100 \mu \mathrm{M}$ NOC18, $20 \mu \mathrm{M}$ Rp-cAMPS (Calbiochem), or $20 \mu \mathrm{M}$ Sp-cAMPS (Calbiochem). A higher concentration ( $40 \mu \mathrm{M})$ of Sp-cAMPS was also used in Figure 7.

Immunocytochemistry. To normalize the antigen distribution by growth cone thickness, a fixable analog of Alexa 594-conjugated dextran (molecular weight, 10,000; Invitrogen) was preintroduced into chick DRG neurons by trituration loading (Nishimura et al., 2003). The cultured neurons were fixed in fixation buffer ( $80 \mathrm{~mm}$ Na-PIPES, pH 6.9, 1 mм $\mathrm{MgCl}_{2}, 1$ mм EGTA, 1 mm GTP, $3 \%$ sucrose, $0.1 \%$ glutaraldehyde, and $4 \%$ formaldehyde) for $30 \mathrm{~min}$ at $37^{\circ} \mathrm{C}$, permeabilized with $0.1 \%$ Triton X-100 for $60 \mathrm{~min}$, and then incubated with mouse anti-nNOS monoclonal antibody (1:10, A-11; Santa Cruz Biotechnology) or rabbit anti-RyRs polyclonal antibody (1:5000, anti-C2) (Kuwajima et al., 1992) overnight at $4^{\circ} \mathrm{C}$. Primary antibody binding was visualized with Alexa 488-conjugated goat anti-mouse or anti-rabbit IgG secondary antibody (1:200; Invitrogen). Ratiometric images were obtained by dividing Alexa 488 signals by Alexa 594 signals using AquaCosmos.

Imaging of FLIP-induced $\mathrm{Ca}^{2+}$ signals. FLIP-induced $\mathrm{Ca}^{2+}$ signals in a growth cone were visualized by simultaneous and ratiometric imaging of two fluorescent $\mathrm{Ca}^{2+}$ indicators, Oregon Green 488 BAPTA-1 (OGB-1) (Invitrogen) and Fura Red (FR) (Invitrogen), which rules out the artifactual fluorescence changes attributable to the growth cone movements and photobleaching of $\mathrm{Ca}^{2+}$ indicators (Gomez et al., 2001; Henley et al., 2004). As $\mathrm{Ca}^{2+}$ concentrations elevate, the fluorescence emission of OGB-1 increases and that of FR decreases. Cultured chick DRG neurons were loaded simultaneously with $2 \mu \mathrm{M}$ OGB-1-AM, $2 \mu \mathrm{M}$ FR-AM, and 2 $\mu \mathrm{M}$ NP-EGTA-AM in the presence of $0.0025 \%$ Cremophor EL (Sigma) for $30 \mathrm{~min}$, followed by a wash. The neurons were postincubated for $>30$ min and then observed under an inverted microscope (IX71) equipped with a $100 \times$ objective (UPLSAPO) and a CCD camera (ORCA-ER). OGB-1 and FR were simultaneously excited with a $75 \mathrm{~W}$ xenon lamp using a bandpass filter (460-495BP; Olympus) and a dichroic mirror (72100bs; Chroma Technology). The OGB-1 and FR emissions were split by a dichroic mirror (DM590LP; Hamamatsu Photonics) equipped in an emission splitter (W-view; Hamamatsu Photonics). The split OGB-1 and FR emissions were collected through a bandpass filter (535AF45; Omega) and a long-pass filter (BA610IF; Olympus), respectively. The images of OGB-1 and FR were simultaneously acquired every $22.1 \mathrm{~ms}$ at an exposure of $10.2 \mathrm{~ms}$ with CCD binning set at $8 \times 8$. For FLIP of NP-EGTA, five laser pulses (a pulse width of $5 \mathrm{~ns}$ ) were shot onto a growth cone at $442 \mathrm{~ms}$ intervals, which corresponded to one laser pulse per 20 frames of $\mathrm{Ca}^{2+}$ imaging. The laser-shot timing was controlled by AquaCosmos and an electronic stimulator (Nihon Koden) such that a camera exposure was initiated $0.9 \mathrm{~ms}$ after the laser shot. This interval was sufficiently long to prevent laser-induced artifacts from affecting $\mathrm{Ca}^{2+}$ imaging (Ooashi et al., 2005).

For quantitative analyses, a region of interest (ROI) $(2.6 \mu \mathrm{m}$ diameter circular zone) was positioned within a growth cone such that the ROI was centered by the FLIP site. After background subtraction, fluorescence intensities $(F)$ of OGB-1 and FR were averaged within the ROI. Relative fluorescence over the basal fluorescence $\left(F / F_{0}\right)$ was calculated individually for OGB- 1 and FR channels. Here, $F_{0}$ is a mean of nine consecutive $F$ values taken from 0 to $176.6 \mathrm{~ms}$ (before the first laser shot). The $F / F_{0}$ values for OGB-1 and FR channels were designated as $R_{\mathrm{OGB}-1}$ and $R_{\mathrm{FR}}$, respectively. Changes in cytosolic $\mathrm{Ca}^{2+}$ levels were expressed as $\Delta\left(R_{\mathrm{OGB}-1} /\right.$ $\left.R_{\mathrm{FR}}\right)$, where $\Delta\left(R_{\mathrm{OGB}-1} / R_{\mathrm{FR}}\right)=R_{\mathrm{OGB}-1} / R_{\mathrm{FR}}-1$. Positive and negative $\Delta\left(R_{\mathrm{OGB}-1} / R_{\mathrm{FR}}\right)$ values indicate that $\mathrm{Ca}^{2+}$ levels increase and decrease 
with respect to the basal $\mathrm{Ca}^{2+}$ level, respectively, in which the basal $\mathrm{Ca}^{2+}$ level is the mean of nine frames taken from 0 to $176.6 \mathrm{~ms}$ (before the first laser shot). The effect of pharmacological agents on FLIP-induced $\mathrm{Ca}^{2+}$ elevations was evaluated by comparing the amplitude of $\Delta\left(R_{\mathrm{OGB}-1} / R_{\mathrm{FR}}\right)$ spikes before and after $5 \mathrm{~min}$ treatment with the drugs in the same growth cone (see Fig. 5). The drug-induced changes in the amplitude of $\Delta\left(R_{\mathrm{OGB}-1} / R_{\mathrm{FR}}\right)$ spikes were expressed as $\Delta R^{\prime}{ }_{\text {after }} / \Delta R_{\text {before, where }}^{\prime} \Delta R_{\text {before }}^{\prime}$ and $\Delta R^{\prime}{ }_{\text {after }}$ indicate the mean of five peak $\Delta\left(R_{\mathrm{OGB}-1} / R_{\mathrm{FR}}\right)$ values induced by five laser pulses before and after the drug treatment, respectively.

Statistics. Data were expressed as the mean \pm SEM. Statistical analyses were performed using Prism version 4.03 software (GraphPad Software). $p$ values $<0.05$ were judged statistically significant.

\section{Results \\ CAMs regulate NO and cGMP levels in DRG neurons}

To test whether extracellular adhesive environments influence the activity of the NO-cGMP pathway, we cultured chick DRG neurons on laminin or L1 substrate and measured intracellular levels of $\mathrm{NO}$ and cGMP (Fig. 1). Intracellular NO production was quantified using DAF-FM, a fluorescent NO indicator, that traps NO to yield a highly fluorescent triazole compound in a cell (Kojima et al., 1999). Because the reaction of NO with DAF-FM is irreversible, the intensity of DAF-FM fluorescence corresponds to cumulative $\mathrm{NO}$ production at the time of image acquisition. The intensity of DAF-FM fluorescence was higher in growth cones on laminin than those on L1, and the difference was negated by pretreating neurons with PTIO, an NO scavenger (Fig. $1 A-C$ ). Similarly, neuronal cell bodies on laminin showed higher DAF-FM fluorescence than those on L1, and the difference was negated by PTIO (Fig. 1D). Treatment with NOC18, an NO donor, markedly increased DAF-FM fluorescence in neuronal cell bodies on L1: $1.00 \pm 0.14$ in untreated neurons ( $n=54$ cells) versus $1.98 \pm 0.26$ in NOC18treated neurons $(n=53$ cells), in which the mean DAF-FM fluorescence intensity in untreated neurons was normalized to 1.00. The difference is statistically significant at the $p<0.01$ level (unpaired $t$ test).

Next we measured intracellular cGMP levels in chick DRG neurons by enzyme immunoassay (Fig. 1E). Concentrations of cGMP in neurons on laminin were higher than those on L1. PTIO treatment decreased cGMP concentrations in neurons on laminin, suggesting that laminin increases cGMP levels by stimulating NO production. We also showed that a pharmacological elevation of NO with NOC18 markedly increased cGMP levels in neurons on L1: $1.00 \pm 0.11$ in untreated neurons versus $3.45 \pm 0.36$ in NOC18-treated neurons $(n=3$ wells each), in which the mean cGMP concentration in untreated neurons was normalized to 1.00. The difference is statistically significant at the $p<0.01$ level (unpaired $t$ test).
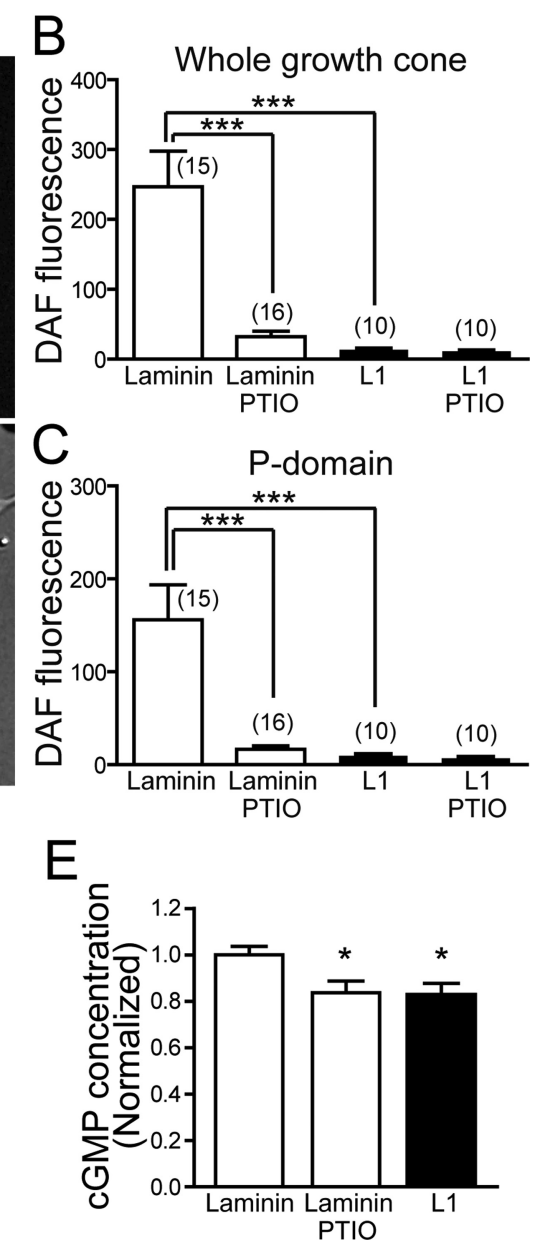

Figure 1. CAMs regulate NO and cGMP levels in neurons. $\boldsymbol{A}$, Fluorescence (top row) and corresponding differential interference contrast (DIC) (bottom row) images of chick DRG growth cones on laminin (left column) and L1 (right column) stained with the of comparison test. $\boldsymbol{E}$, Concentrations of CGMP in chick DRG neurons on laminin (open bars) or L1 (filled bar) in the absence or presence of PTIO were measured by enzyme immunoassay. Bars represent normalized cGMP concentrations. * $p<0.05$ versus laminin, Dunnett's multiple comparison test ( $n=12$ wells each).

These results indicate that intracellular levels of $\mathrm{NO}$ and cGMP are influenced by extracellular substrates and conform to the concept that NO increases cGMP levels by activating sGC.

\section{The NO-cGMP pathway controls $\mathrm{Ca}^{2+}$-induced growth cone turning}

Next we examined whether the NO-cGMP pathway controls the directional polarity of $\mathrm{Ca}^{2+}$-mediated growth cone guidance (Fig. 2). Growth cone turning was triggered by producing spatially restricted $\mathrm{Ca}^{2+}$ elevations in the growth cone by FLIP of a caged $\mathrm{Ca}^{2+}$ compound, NP-EGTA compounded with $\mathrm{Ca}^{2+}$ in the cytosol (Zheng, 2000; Ooashi et al., 2005). This type of experimental manipulation has been used extensively to study causal relationships between $\mathrm{Ca}^{2+}$ and other signals in axon growth and guidance (Robles et al., 2003; Wen et al., 2004; Tojima et al., 2007). Consistent with our previous report (Ooashi et al., 2005), repetitive FLIP (3 s intervals) of NP-EGTA on one side of a chick DRG growth cone induced bidirectional turning depending on culture substrates: turning away from the laser-shot side (referred 


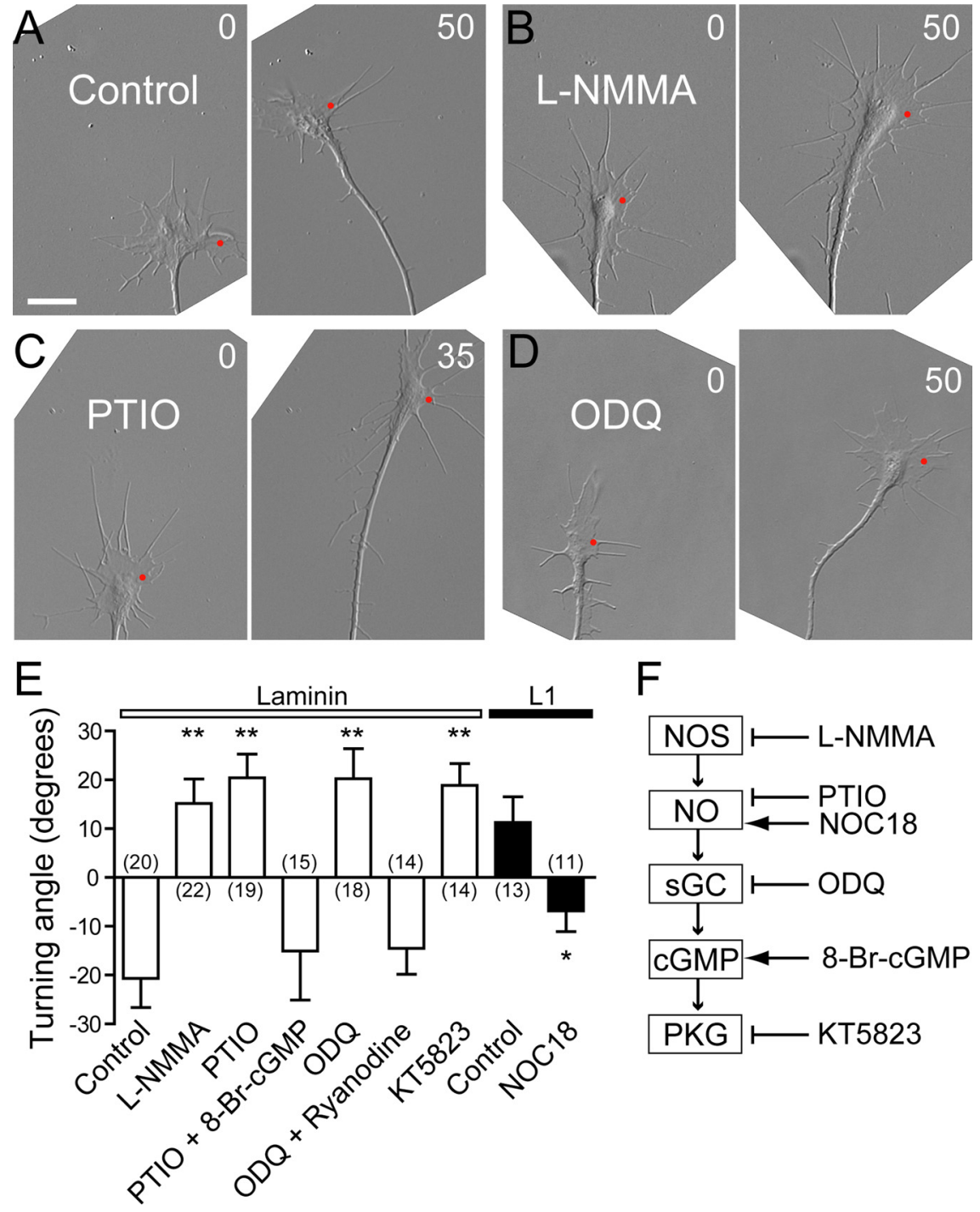

Figure 2. The NO- $C$ GMP pathway controls the direction of $\mathrm{Ca}^{2+}$-induced growth cone turning. $\boldsymbol{A}-\boldsymbol{D}$, Time-lapse DIC images of chick DRG growth cones on laminin in the absence $\left(\boldsymbol{A}\right.$, control) or presence of L-NMMA $(\boldsymbol{B})$, PTIO ( $\boldsymbol{C}$, or ODQ (D). Focal $\mathrm{Ca}^{2+}$ signals were generated on one side of the growth cone by repetitive FLIP ( 3 intervals) indicated here by red spots. Digits represent minutes after the onset of repetitive FLIP. Scale bar, $10 \mu \mathrm{m}$. E, Turning angles of growth cones on laminin (open bars) or L1 (filled bars) induced by focal $\mathrm{Ca}^{2+}$ signals in the absence (control) or presence of the indicated drugs. Turning angles with positive and negative values indicate attraction and repulsion, respectively. Numbers in parentheses indicate the total number of growth cones examined. ${ }^{* *} p<0.01$ versus laminin control, Dunnett's multiple comparison test. ${ }^{*} p<0.05$ versus $\mathrm{L} 1$ control, unpaired $t$ test. $\boldsymbol{F}$, Pharmacological agents used in this study. L-NMMA, A NOS inhibitor; PTIO, an NO scavenger; NOC18, an NO donor; ODQ, an SGC inhibitor; 8-Br-cGMP, a cGMP analog; KT5823, a PKG inhibitor.

to as repulsion) on laminin or turning toward the laser-shot side (referred to as attraction) on $\mathrm{L} 1$ (Fig. $2 \mathrm{~A}, \mathrm{E})$. The $\mathrm{Ca}^{2+}$-induced repulsion on laminin was converted to attraction when $\mathrm{NO}$ signaling was blocked by bath application of either L-NMMA, an inhibitor of NOS, or PTIO (Fig. $2 B, C, E$ ). This conversion was rescued by simultaneous treatment with 8 -Br-cGMP, an analog of cGMP (Fig. 2 E, PTIO + 8-Br-cGMP), consistent with the idea that cGMP is a downstream effector of NO. The involvement of cGMP-associated signals in the regulation of growth cone turning was further tested using additional drugs: ODQ, an inhibitor of sGC, and KT5823, an inhibitor of PKG. Bath application of either of these two drugs converted repulsion to attraction on laminin (Fig. $2 \mathrm{D}, E$ ). These results indicate that $\mathrm{Ca}^{2+}$-induced repulsion on laminin involves the activity of the NOS-NO-sGCcGMP-PKG pathway and that inactivation of this pathway con- verts the $\mathrm{Ca}^{2+}$-induced repulsion to attraction. Based on the previous finding that the turning direction of growth cones depends on the occurrence of CICR through RyRs (Hong et al., 2000; Ooashi et al., 2005), we tested whether CICR is involved in the CGMP-mediated regulation of growth cone turning. In the presence of a high dose $(100 \mu \mathrm{M})$ of ryanodine that traps RyRs in the closed state (Zucchi and RoncaTestoni, 1997), ODQ could not convert repulsion to attraction (Fig. 2E, ODQ + Ryanodine), suggesting that sGC-produced cGMP controls the turning direction via modulating RyR-mediated CICR. We also tested whether increasing NO levels with NOC18 modulates growth cone turning responses. A previous report showed that treating Helisoma neurons with 1-hydroxy2-oxo-3-(N-methyl-3-aminopropyl)-3methyl-1-triazene (NOC7), another NO donor, inhibits neurite extension (Trimm and Rehder, 2004). In our experiments, however, bath application of NOC18 to chick DRG neuronal cultures had no detectable effect on the rate of growth cone migration (data not shown). Repetitive FLIP of NP-EGTA on one side of a growth cone induced attractive turning on L1 (Fig. $2 E$ ). This attraction was converted to repulsion when NO levels were elevated by treatment with NOC18. Taken collectively, these results indicate that the NO-cGMP pathway plays a key role in switching the direction of $\mathrm{Ca}^{2+}$-induced growth cone turning probably by modulating the activity of RyRs.

It is well known that cAMP switches the directional polarity of growth cone turning: higher and lower cAMP levels favor attraction and repulsion, respectively (Ming et al., 1997; Song et al., 1997, 1998; Ooashi et al., 2005). To test for the possibility that the NO-cGMP pathway converts the turning direction via changing cAMP levels, we measured cAMP levels in neurons by enzyme immunoassay. Scavenging NO with PTIO had no effect on cAMP concentrations in neurons on laminin: $1.00 \pm 0.05$ in untreated neurons versus $1.03 \pm 0.07$ in PTIO-treated neurons $(n=21$ wells each), in which the mean cAMP concentration in untreated neurons was normalized to 1.00. Similarly, elevating NO levels with NOC18 had no effect on cAMP concentrations in neurons on L1: $1.00 \pm 0.04$ in untreated neurons versus $0.96 \pm$ 0.04 in NOC18-treated neurons ( $n=21$ wells each). These results indicate that the NO-cGMP pathway switches the direction of growth cone turning independently of cAMP.

\section{NO-mediated switching of growth cone turning direction depends on nNOS}

There are three isoforms of NOS: nNOS, endothelial NOS (eNOS), and inducible NOS (iNOS). Previous reports showed that developing DRG neurons in vivo strongly express nNOS 
proteins (Qian et al., 1996; Thippeswamy et al., 2005). We examined the subcellular distribution of nNOS proteins in cultured DRG growth cones (Fig. 3). To normalize nNOS immunofluorescence by the growth cone thickness, the growth cone cytosol was colabeled with Alexa 594-conjugated dextran. The spatial profile of the ratio of nNOS immunofluorescence to Alexa 594dextran fluorescence represented that nNOS proteins were distributed over the entire region of growth cones on laminin and L1 (Fig. 3A). We also showed the homogenous distribution of RyR proteins in growth cones (Fig. 3B).

To test whether nNOS is responsible for producing NO that controls axon guidance, we analyzed the turning behaviors of DRG growth cones derived from an nNOS-gene knock-out mouse line (Huang et al., 1993) (Fig. 4). On the laminin substrate, asymmetric $\mathrm{Ca}^{2+}$ signals generated by repetitive FLIP caused attractive turning of nNOS-deficient growth cones, whereas wild-type growth cones exhibited repulsive turning (Fig. $4 A, B, D$ ). The attraction of nNOS-deficient growth cones was converted to repulsion by NOC18 treatment (Fig. 4C,D), suggesting that the lack of NO production is responsible for the altered turning behavior caused by nNOS deficiency. These data indicate that nNOS in neurons is involved in the regulation of growth cone turning induced by $\mathrm{Ca}^{2+}$ signals.

\section{RyR-mediated CICR is regulated by the} NO-cGMP pathway

With $\mathrm{Ca}^{2+}$ imaging combined with FLIP of caged $\mathrm{Ca}^{2+}$, we previously established an experimental method to determine whether CICR components are included in FLIP-induced $\mathrm{Ca}^{2+}$ elevations. This method has been used successfully to demonstrate that the occurrence of CICR depends on the activity of cAMP-PKA pathway (Ooashi et al., 2005): FLIP of NPEGTA triggers CICR when cAMP levels are high, e.g., in growth cones on L1, whereas FLIP of NP-EGTA fails to trigger CICR when cAMP levels are low, e.g., in growth cones on laminin. In the present study, we used this method with some modifications to test whether CICR is regulated downstream of the NO-cGMP pathway (Fig. 5). We generated five $\mathrm{Ca}^{2+}$ transients by five repetitive FLIP of NPEGTA in a chick DRG growth cone (Fig. $5 A, B)$. The FLIP-induced $\mathrm{Ca}^{2+}$ transients were visualized by simultaneous and ratiometric imaging of two fluorescent $\mathrm{Ca}^{2+}$ indicators, OGB-1 and FR (for details, see Materials and Methods). These $\mathrm{Ca}^{2+}$ ele-
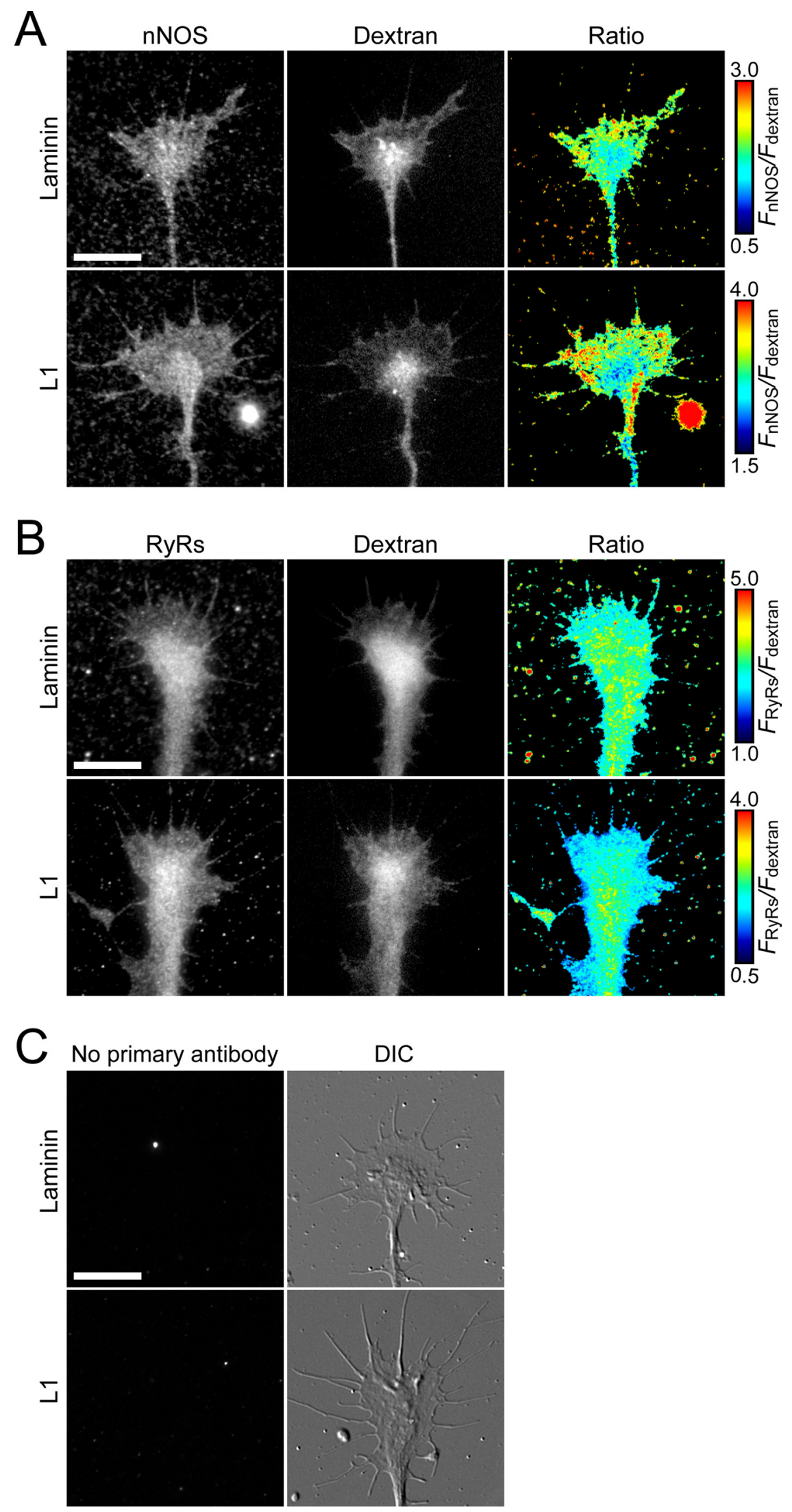

Figure 3. $\mathrm{nNOS}$ and RyRs are distributed over the entire region of the growth cone. $\boldsymbol{A}, \boldsymbol{B}, \mathrm{nNOS}(\boldsymbol{A})$ or RyR $(\boldsymbol{B})$ immunofluorescence in chick DRG growth cones on laminin or L1. Alexa 594-conjugated dextran that had been introduced into growth cones was used as a measure of cytoplasm thickness. The pseudocolor images show the ratio of immunofluorescence $\left(F_{\mathrm{nNOS}}\right.$ or $\left.F_{\mathrm{RyRs}}\right)$ to Alexa 594 fluorescence $\left(F_{\text {dextran }}\right)$. C, Negative-control immunocytochemistry in which the primary antibody was omitted. Corresponding DIC images are also shown. Scale bars, $10 \mu \mathrm{m}$. 

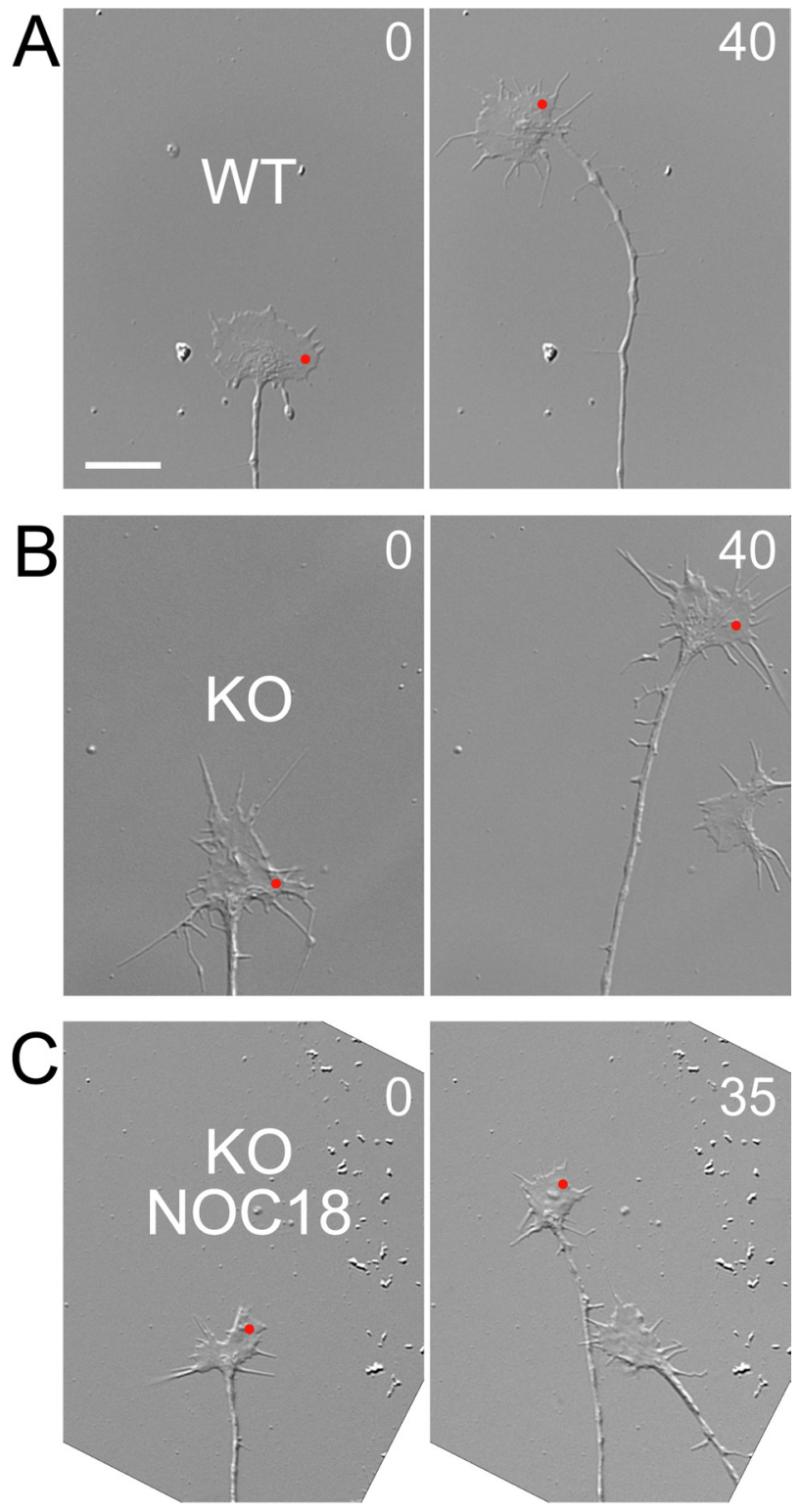

$D$

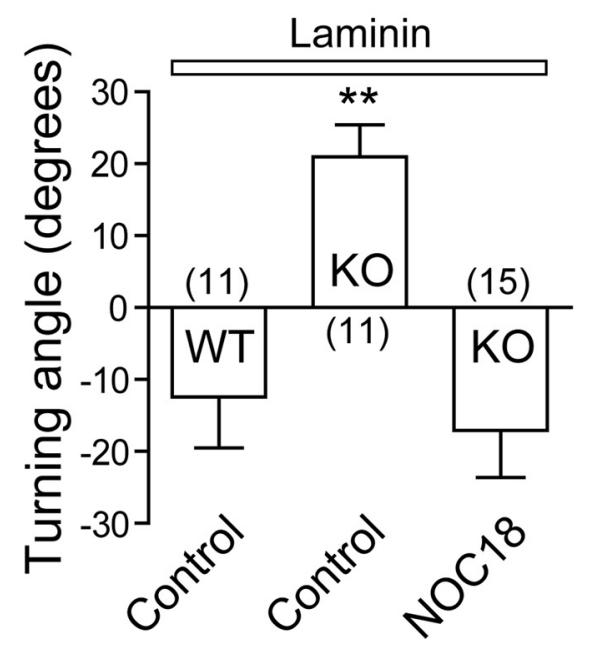

Figure 4. nNOS is involved in the regulation of $\mathrm{Ca}^{2+}$-induced growth cone turning. $\boldsymbol{A}-\mathrm{C}$, Time-lapse DIC images of DRG growth cones derived from wild-type (WT; $A$ ) or nNOS knock-out $(\mathrm{KO} ; \boldsymbol{B}, \boldsymbol{C})$ mice on laminin in the absence $(\boldsymbol{A}, \boldsymbol{B})$ or presence $(\boldsymbol{C})$ of NOC18. Focal $\mathrm{Ca}^{2+}$ signals were generated on one side of the growth cone by repetitive FLIP ( 3 sintervals) at red spots. Digits represent minutes after the onset of repetitive FLIP. Scale bar, $10 \mu \mathrm{m}$. D, Turning angles vations should be the sum of $\mathrm{Ca}^{2+}$ liberated from NP-EGTA and, if applicable, CICR. To dissect these two different $\mathrm{Ca}^{2+}$ sources, we compared the amplitude of $\mathrm{Ca}^{2+}$ elevations in the same growth cones before and after pharmacological treatments. Consistent with our previous study (Ooashi et al., 2005), the mean amplitude of five $\mathrm{Ca}^{2+}$ elevations generated in growth cones on laminin was increased after 5 min treatment of these growth cones with Sp-cAMPS, a cAMP agonist (Fig. 5C). It was already shown that the Sp-cAMPS-induced increase in $\mathrm{Ca}^{2+}$-signal amplitude was abolished by simultaneous treatment with a high dose of ryanodine, indicating that Sp-cAMPS facilitates an additional increase in $\mathrm{Ca}^{2+}$ via RyRs, i.e., CICR (Ooashi et al., 2005). Similarly, FLIP-induced $\mathrm{Ca}^{2+}$ elevations on laminin were enhanced after treatment with L-NMMA, ODQ, or KT5823 (Fig. $5 A, C)$. The enhancement of $\mathrm{Ca}^{2+}$-signal amplitude by these drugs was abolished by simultaneous treatment with a high dose of ryanodine (Fig. 5C, L-NMMA + Ryanodine, ODQ + Ryanodine, KT5823 + Ryanodine), indicating that inhibition of the NO-cGMP-PKG pathway augments $\mathrm{Ca}^{2+}$ signals by facilitating RyR-mediated CICR. Conversely, FLIP-induced $\mathrm{Ca}^{2+}$ elevations on L1 included CICR components that were blocked by inactivating RyRs (Fig. 5D). Elevating NO with NOC18 also suppressed the amplitude of FLIP-induced $\mathrm{Ca}^{2+}$ elevations on L1 (Fig. $5 B, D$ ). These results are consistent with the idea that the NOcGMP-PKG pathway is a negative regulator of RyR-mediated CICR: high NO-cGMP activities prevent CICR, and low NOcGMP activities allow the occurrence of CICR.

\section{NO counteracts the effect of cAMP on growth cone turning}

A previous report demonstrated that the polarity of growth cone turning induced by an extracellular gradient of netrin-1 is determined by relative activities of cAMP to cGMP, i.e., higher and lower ratio leads growth cone attraction and repulsion, respectively (Nishiyama et al., 2003). Based on this finding, we examined the counteraction between the NO-cGMP and cAMP pathways in determining the direction of growth cone turning induced by asymmetric $\mathrm{Ca}^{2+}$ signals (Fig. 6). When the NOcGMP and cAMP pathways were simultaneously activated with Sp-cAMPS and NOC18 on laminin, repetitive FLIP of NP-EGTA on one side of a chick DRG growth cone triggered no turning response (Fig. $6 \mathrm{~A}-\mathrm{C}$ ), indicating the antagonizing activities of the two pathways. We also assessed growth cone turning when the two pathways were simultaneously inactivated. In the presence of both PTIO and Rp-cAMPS, a cAMP antagonist, the growth cone on laminin exhibited no turning response with respect to asymmetric $\mathrm{Ca}^{2+}$ signals $\left(1.88 \pm 2.92^{\circ} ; n=12\right.$ growth cones), whereas the control growth cone treated with Rp-cAMPS alone exhibited repulsive turning $\left(-12.69 \pm 5.99^{\circ} ; n=12\right.$ growth cones). The difference is statistically significant at the $p<$ 0.05 level (unpaired $t$ test). This antagonism between PTIO and Rp-cAMPS was also confirmed in growth cones on L1 (Fig. 6D). Together, these results are consistent with the notion that relative, not absolute, activities of the NO-cGMP and cAMP pathways are the critical determinant of the directional polarity of growth cone guidance.

of growth cones on laminin induced by focal $\mathrm{Ca}^{2+}$ signals in the absence (control) or presence of NOC18. Turning angles with positive and negative values indicate attraction and repulsion, respectively. Numbers in parentheses indicate the total number of growth cones examined. ${ }^{* *} p<0.01$ versus wild-type control, Dunnett's multiple comparison test. 
A
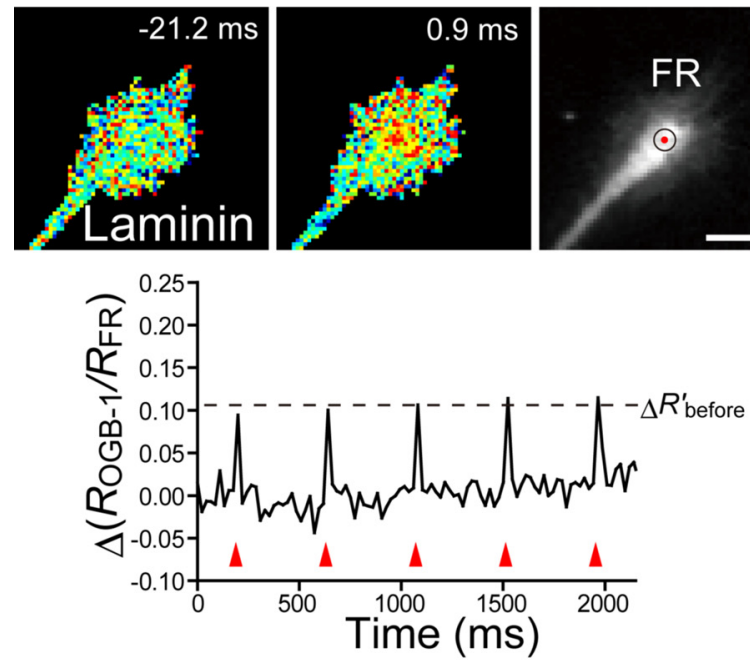

B

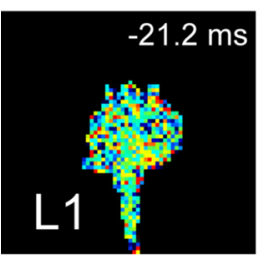

Before
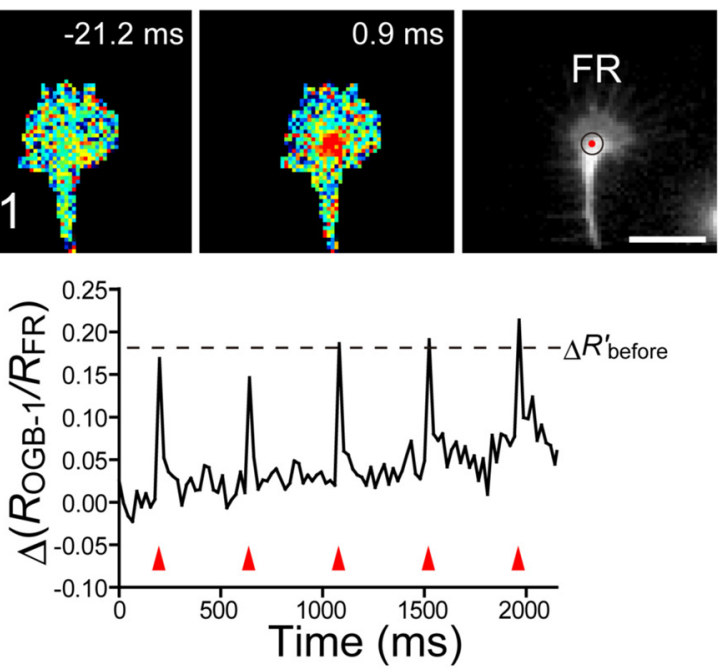

C

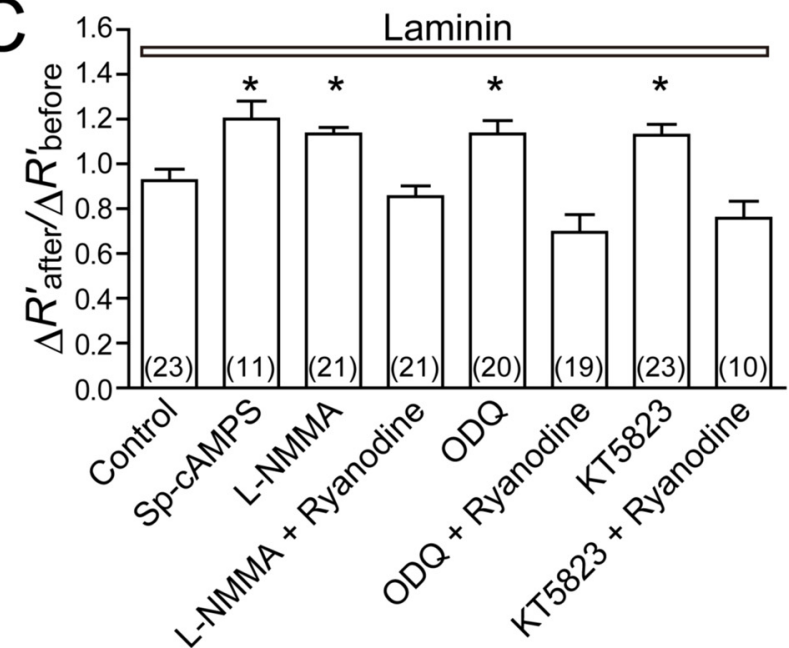

After L-NMMA treatment
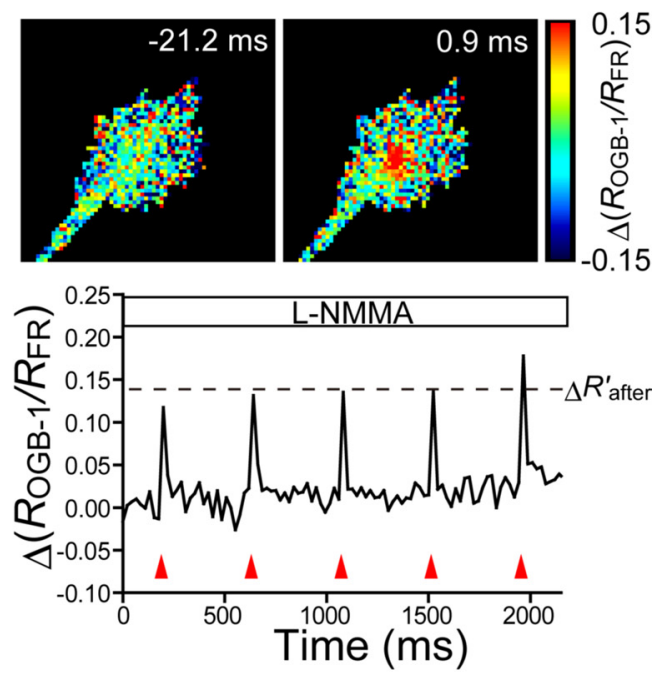

After NOC18 treatment
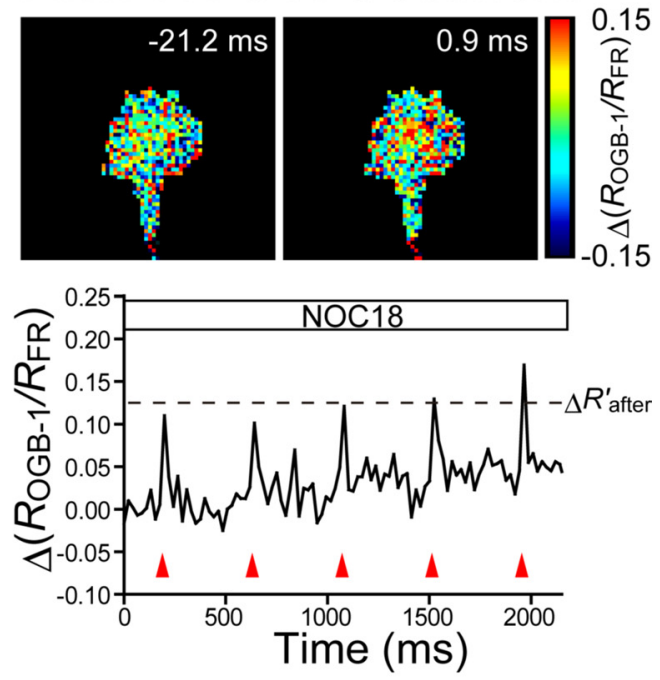

D

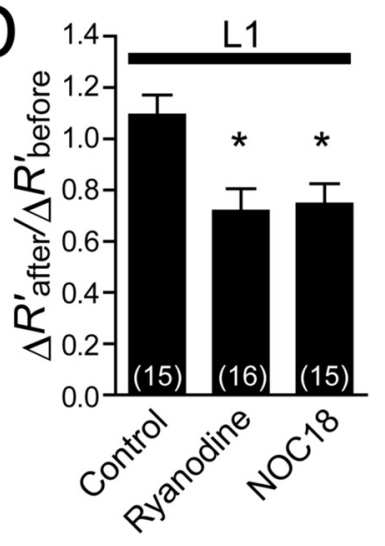

Figure 5. The NO- cGMP pathway regulates RyR-mediated CICR in growth cones. $\boldsymbol{A}, \boldsymbol{B}$, Chick DRG growth cones were loaded with NP-EGTA and two Ca ${ }^{2+}$ indicators, 0 GB-1 and FR. Shown are a growth cone on laminin with L-NMMA treatment $(\boldsymbol{A})$ and a growth cone on $\mathrm{L} 1$ with NOC18 treatment $(\boldsymbol{B})$. FLIP-induced $\mathrm{Ca}^{2+}$ elevations in the growth cones were quantified by ratiometric $C \mathrm{a}^{2+}$ imaging. Before (left side) and after (right side) 5 min treatment with the indicated drugs, the $\mathrm{Ca}^{2+}$ signals were analyzed in the same growth cones under the same FLIP conditions. The black and white images show fluorescence intensities of FR. The red spots and the black circles in the FR images represent, respectively, the sites of laser irradiation and ROIs used to quantify $\mathrm{Ca}^{2+}{ }_{\text {-signal }}$ amplitude. The ROI was defined as a 2.6- $\mu$ m-diameter zone centered by the FLIP site. The pseudocolor time-lapse images show changes in the ratio of OGB-1 to FR (Figure legend continues.) 

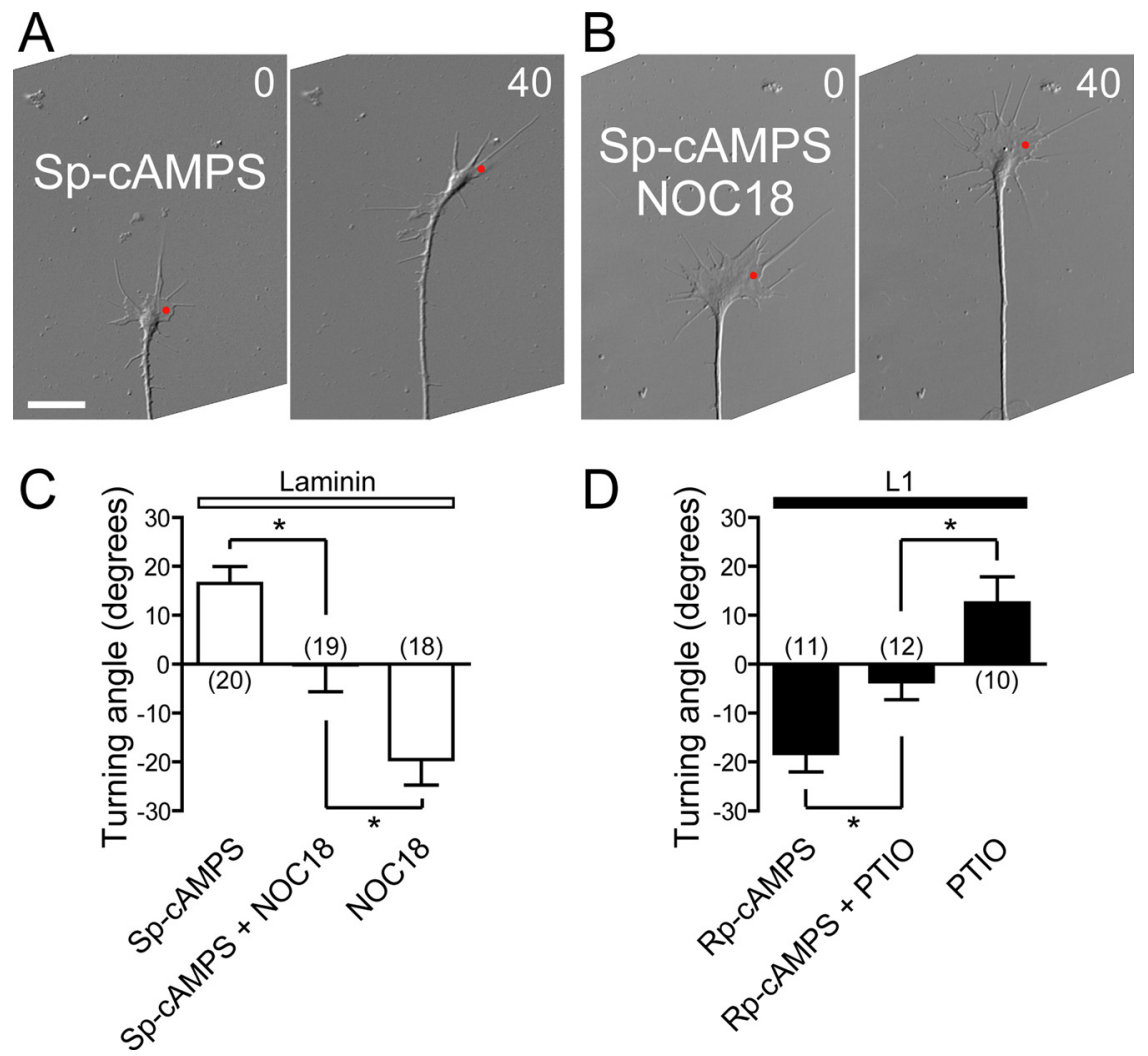

Figure 6. Counteractive effects of $\mathrm{NO}$ and CAMP on $\mathrm{Ca}^{2+}$-induced growth cone turning. $A, B$, Time-lapse DIC images of chick DRG growth cones on laminin in the presence of Sp-CAMPS alone $(\boldsymbol{A})$ or Sp-CAMPS and NOC18 $(\boldsymbol{B})$. Focal $\mathrm{Ca}^{2+}$ signals were generated on one side of the growth cone by repetitive FLIP ( 3 sintervals) indicated by red spots. Digits represent minutes after the onset of repetitive FLIP. Scale bar, $10 \mu \mathrm{m}$. C, D, Turning angles of growth cones on laminin $(C)$ or L1 (D) induced by focal $\mathrm{Ca}^{2+}$ signals in the presence of the indicated drugs. Turning angles with positive and negative values indicate attraction and repulsion, respectively. Numbers in parentheses indicate the total number of growth cones examined. ${ }^{*} p<0.05$, Bonferroni's multiple comparison test.

\section{The NO-cGMP pathway is involved in NT-4-induced growth cone repulsion}

Next we examined whether the NO-cGMP pathway is involved in growth cone guidance induced by an extracellular physiological cue (Fig. 7). A previous report showed that local application of NT-4, a neurotrophic factor that activates the TrkB tyrosine kinase receptor, through a glass pipette to a DRG growth cone induces growth cone collapse and axon growth inhibition (Paves and Saarma, 1997). The possibility that NT-4 acts as a guidance cue was tested by generating an extracellular gradient of NT-4 across a chick DRG growth cone. We found that the NT-4 gradient repelled the growth cones on laminin (Fig. $7 A, D$ ). This NT4 -induced repulsion was abolished by pretreatment with a membrane-permeable $\mathrm{Ca}^{2+}$ chelator, BAPTA-AM (Fig. 7D). Us-

\section{$\leftarrow$}

(Figure legend continued.) fluorescence intensities $\left[\Delta\left(R_{0 \mathrm{~GB}-1} / R_{\mathrm{FR}}\right)\right.$, where $\left.R=F / F_{0}\right]$ (for details, see Materials and Methods). Digits in the pseudocolor images represent milliseconds after single FLIP. Scale bars, $10 \mu \mathrm{m}$. The graphs show time course changes in $\Delta\left(R_{0 \mathrm{~GB}-1} / R_{\mathrm{FR}}\right)$ values averaged within the ROl. Note that five laser pulses (red arrowheads) triggered five $\mathrm{Ca}^{2+}$ elevations $\left[\Delta\left(R_{0 \mathrm{~GB}-1} / R_{\mathrm{FR}}\right)\right.$ spikes]. The dashed lines indicate the mean of the five peak $\Delta\left(R_{0 \mathrm{~GB}-}\right.$ $\left.1 / R_{F R}\right)$ values before the drug treatment $\left(\Delta R^{\prime}\right.$ before $)$ or the mean of those after the drug treatment $\left(\Delta R^{\prime}\right.$ after $) \cdot C, D$, The effects of the indicated drugs on FLIP-induced $\mathrm{Ca}^{2+}$ elevations were evaluated in growth cones on laminin $(\boldsymbol{C})$ or $L 1(\boldsymbol{D})$. "Control" indicates that drug treatment was omitted. $\Delta R^{\prime}$ after $/ \Delta R^{\prime}$ before in the $y$-axis represents the relative changes in $\mathrm{Ca}^{2+}$-signal amplitude caused by the drug treatment. Numbers in parentheses indicate the total number of growth cones examined. ${ }^{*} p<0.05$ versus control, Dunnett's multiple comparison test. ing simultaneous and ratiometric imaging of OGB-1 and FR, we also showed that the NT-4 gradient evoked asymmetric $\mathrm{Ca}^{2+}$ signals, with $\mathrm{Ca}^{2+}$ concentrations higher on the side of the growth cone facing the NT-4 source (supplemental Fig. 1, available at www.jneurosci.org as supplemental material). These results suggest that NT-4 repels the growth cones via asymmetric $\mathrm{Ca}^{2+}$ signals. We then examined whether the NO-cGMP pathway is involved in NT-4-induced repulsion. Bath application of PTIO or ODQ converted the NT-4induced repulsion to attraction (Fig. $7 B$ $D)$, indicating that the activity of $\mathrm{NO}-$ cGMP signaling is a critical determinant of whether NT-4 attracts or repels the growth cones. The effect of cAMP on NT-4induced repulsion was also tested (Fig. $7 D)$. Bath application of Sp-cAMPS (20 $\mu \mathrm{M})$ caused the growth cones to migrate practically straight in the NT-4 gradient. When we applied a higher concentration of Sp-cAMPS $(40 \mu \mathrm{M})$, the growth cones were attracted by the NT-4 gradient. These results are consistent with the notion that the directional polarity of NT-4-induced growth cone turning is controlled by the NO-cGMP and cAMP pathways in a counteractive manner.

\section{Discussion}

This study demonstrates that endogenous NO in DRG neurons is involved in repulsive axon guidance and that extracellular substrates influence the turning responses of the growth cone via modulating the NO-cGMP pathway. The counteraction of the NO-cGMP and cAMP pathways determines the occurrence of RyR-mediated CICR, thereby switching the direction of growth cone turning with respect to asymmetric $\mathrm{Ca}^{2+}$ elevations. Our data also suggest that this regulatory mechanism plays a role in growth cone turning induced by an extracellular gradient of a physiological cue, NT-4.

In our model (Fig. 8), laminin-mediated adhesion activates the NO-cGMP-PKG pathway and inactivates the cAMP-PKA pathway over the entire region of the growth cone, thereby leading RyRs to the inactive state. In response to the reception of an extracellular gradient of guidance cues such as netrin-1, asymmetric $\mathrm{Ca}^{2+}$ elevations across the growth cone are generated via $\mathrm{Ca}^{2+}$ entry through plasma-membrane channels, e.g., transient receptor potential canonical channels (Wang and Poo, 2005). These $\mathrm{Ca}^{2+}$ elevations fail to trigger CICR because RyRs are in the inactive state (Fig. $8 \mathrm{~A}$ ). The asymmetric $\mathrm{Ca}^{2+}$ signals without CICR induce repulsive growth cone turning (Ooashi et al., 2005). In contrast, L1-mediated adhesion leads RyRs to the active state via activating the cAMP-PKA pathway and inactivating the NOcGMP-PKG pathway (Fig. $8 \mathrm{~B}$ ). In this situation, asymmetric $\mathrm{Ca}^{2+}$ signals generated by guidance cues are accompanied by CICR that is sufficient to induce growth cone attraction. Our model implies that, on contact with differing extracellular adhesive environments, growth cones change their turning responses to guidance cues by modulating the efficiency of RyR-mediated 

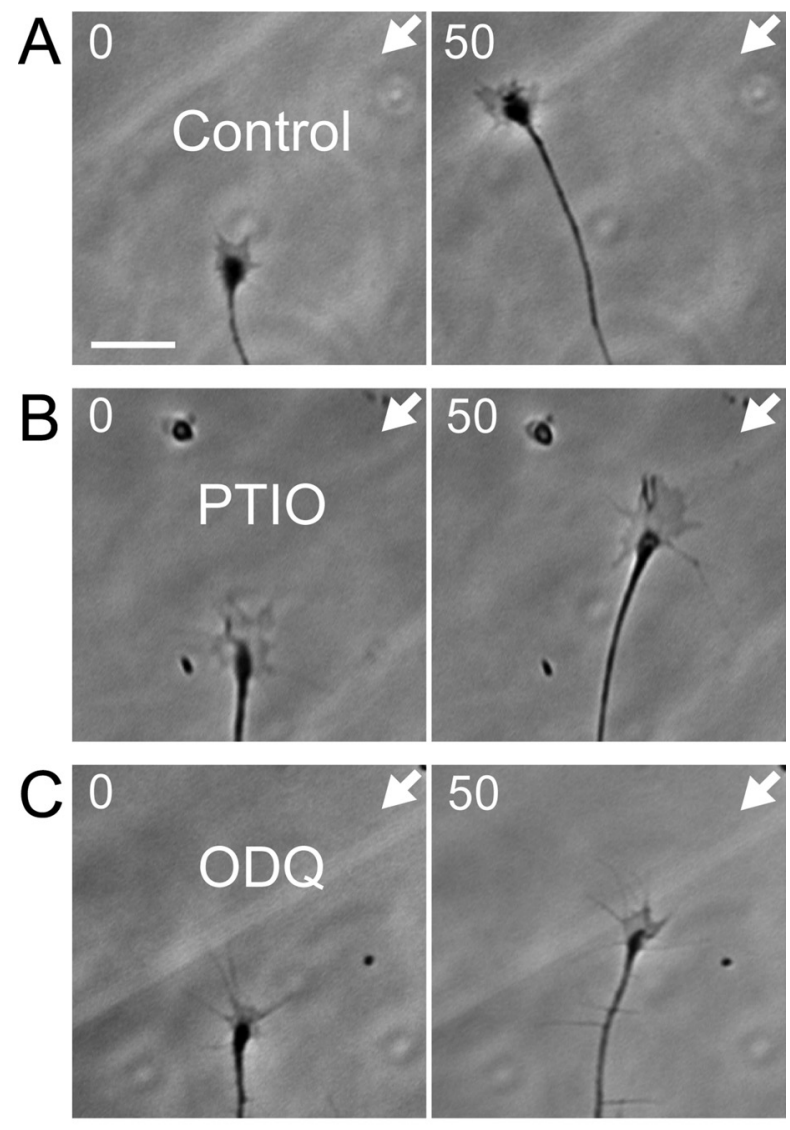

D

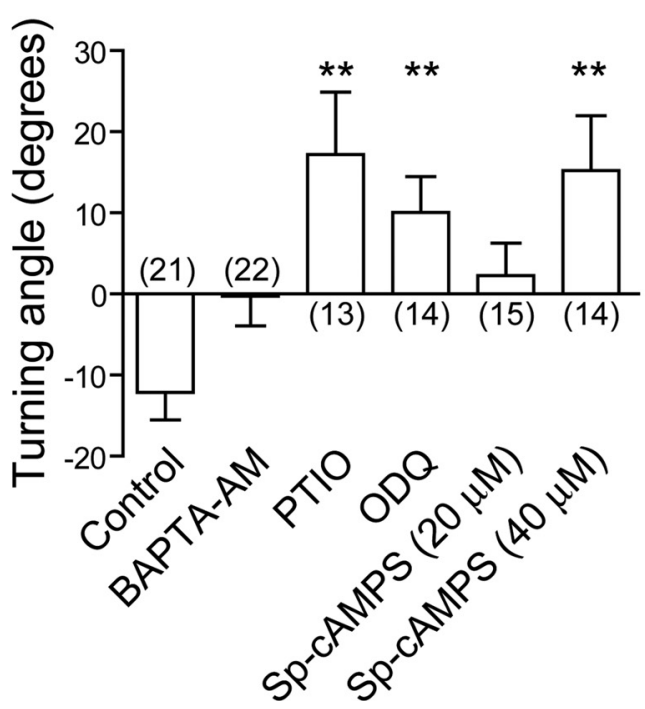

Figure 7. The NO- CGMP pathway controls the direction of NT-4-induced growth cone turning. $\mathbf{A}-\boldsymbol{C}$, Time-lapse phase-contrast images of chick DRG growth cones on laminin that were exposed to an extracellular microscopic gradient of NT-4 in the absence ( $\boldsymbol{A}$, control) or presence of PTIO ( $B$ ) or ODQ ( $C$ ). Digits represent minutes after the onset of NT-4 application. The arrows indicate the direction of an NT-4 gradient. Scale bar, $10 \mu \mathrm{m}$. D, Turning angles of growth cones exposed to the NT-4 gradient. Turning angles with positive and negative values indicate attraction and repulsion, respectively. Numbers in parentheses indicate the total number of growth cones examined. ${ }^{* *} p<0.01$ versus control, Dunnett's multiple comparison test.

CICR via the two counteractive pathways. Such mechanisms may operate in vivo, for example, when a growth cone is migrating through an intermediate target that secretes a guidance cue (Chao et al., 2009). CAMs present in the intermediate target could modulate cyclic nucleotide and $\mathrm{Ca}^{2+}$ signaling in the growth cone, thereby switching its response to the guidance cue from attraction to repulsion.

\section{Regulation of CICR by cyclic nucleotides}

There are several possible signaling cascades that link the NOcGMP pathway with RyRs. Welshhans and Rehder (2007) reported that, downstream of the NO-cGMP-PKG pathway, cADP ribose regulates RyR-mediated CICR to control filopodial dynamics of Helisoma neuronal growth cones. The crosstalk between NO-cGMP and cAMP is also possible. For example, cGMP activates cAMP phosphodiesterases, causing a reduction of cAMP levels (Zaccolo and Movsesian, 2007). However, our enzyme immunoassay data suggested that cAMP concentrations in chick DRG neurons are not regulated by the NO-cGMP pathway. The most likely explanation is that PKA and PKG modulate CICR by phosphorylating RyRs (Suko et al., 1993). Although the physiological relevance of PKG phosphorylation of RyRs remains unknown, PKA phosphorylation potentiates the ion-channel activity of RyRs (Wehrens et al., 2006; Xiao et al., 2006). We propose that RyRs are the site of counteraction of the cAMP-PKA and NO-cGMP$\mathrm{PKG}$ pathways for controlling the occurrence of CICR.

\section{Regulation of NO and cyclic nucleotide levels}

In this study, we show that laminin-mediated adhesion stimulates the production of cGMP via NO. Although intracellular signals responsible for laminin-induced NO elevations remain unclear, there is one report suggesting an extracellular mechanism (Rialas et al., 2000). It showed that, in PC12 cells, bath application of a synthetic peptide, LQVQLSIR, derived from the laminin-1- $\alpha$ globular domain elevates NO levels within seconds of treatment. Because the LQVQLSIR sequence mediates laminin binding to syndecan, a cell surface proteoglycan, it is possible that laminin increases NO levels via binding to syndecan. In contrast to cGMP, DRG growth cones on laminin contain lower levels of cAMP than those on L1 (Ooashi et al., 2005). It was reported that laminin decreases cAMP levels in Xenopus retinal growth cones via YIGSR sequence in the laminin-1- $\beta 1$ chain (Höpker et al., 1999), suggesting that laminin controls the amount of cAMP and cGMP via distinct binding mechanisms on the neuronal surface. Furthermore, Höpker et al. (1999) has demonstrated that the YIGSR peptide applied to developing retina causes axons to be misdirected at the optic nerve head in vivo, stressing the importance of extracellular matrices in growth cone guidance.

\section{Involvement of $\mathrm{NO}$ in growth cone guidance}

Several previous papers showed that NO is implicated in axon guidance. An exogenously applied NO donor converts netrin-1induced attraction to repulsion (Nishiyama et al., 2003) and abolishes Sema3A-induced repulsion (Song et al., 1998). The involvement of endogenous NO has been suggested by Castellani et al. (2002), in which treatment of cortical neurons with 7-nitroindazole, a NOS inhibitor, affects Sema3A-induced axon guidance. In the present study, using nNOS-deficient neurons and various pharmacological agents, we clearly demonstrated that endogenous NO synthesized by nNOS controls axon guidance. The nNOS activity is regulated by direct binding of calmodulin (Su et al., 1995) and phosphorylation by $\mathrm{Ca}^{2+} /$ calmodulindependent protein kinase II (Komeima et al., 2000). Furthermore, nNOS can be proteolytically cleaved by calpain, a $\mathrm{Ca}^{2+}$-dependent protease (Hajimohammadreza et al., 1997). 
A

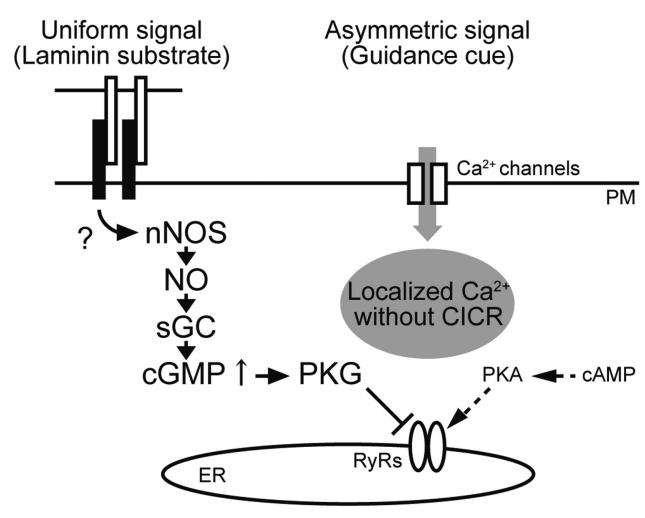

B

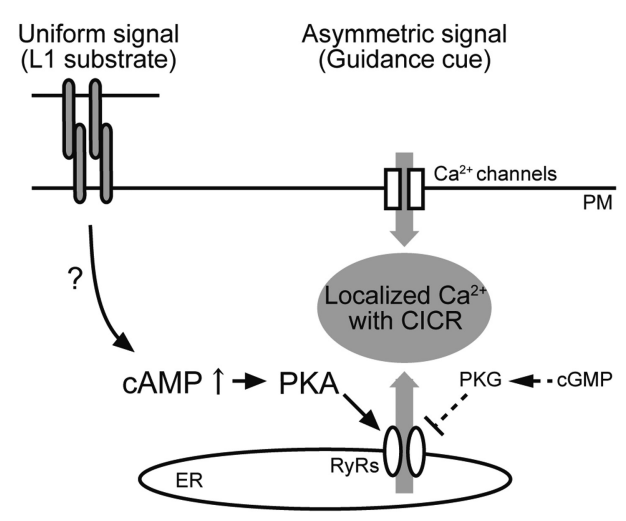

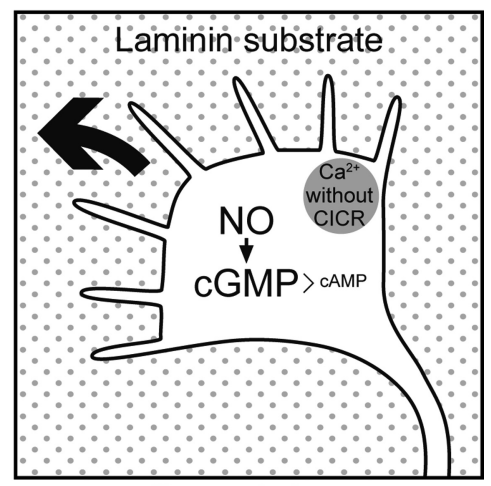

Repulsion

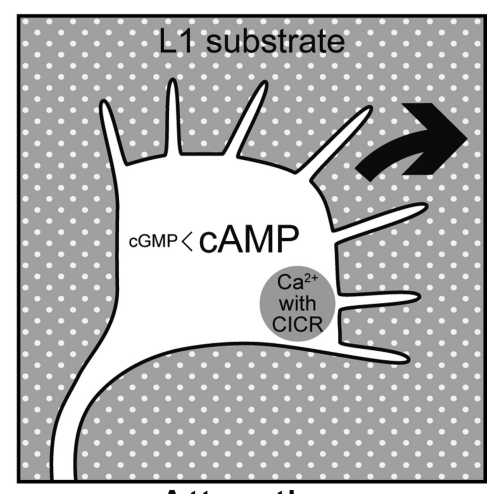

Attraction

Figure 8. A model of bidirectional growth cone turning controlled by cyclic nucleotides and extracellular substrates. $A$, A laminin substrate, which is presented as a uniform signal, stimulates the N0 - cGMP-PKG pathway over the entire spatial extent of the growth cone. The decreased ratio of CAMP to cGMP leads to inactivation of RyRs on the endoplasmic reticulum (ER). After encountering an extracellular gradient of guidance cues such as netrin-1, localized $\mathrm{Ca}^{2+}$ elevations are generated in the growth cone via $\mathrm{Ca}^{2+}$ entry through plasma-membrane (PM) channels. These $\mathrm{Ca}^{2+}$ elevations are not accompanied by CICR because RyRs are in the inactive state. The asymmetric $\mathrm{Ca}^{2+}$ signals without $\mathrm{CICR}$ are sufficient to trigger repulsive turning of the growth cone (right). $\boldsymbol{B}$, An L1 substrate stimulates the CAMP-PKA pathway over the entire spatial extent of the growth cone. The increased ratio of cAMP to cGMP leads RyRs to the active state. $\mathrm{Ca}^{2+}$ entry through PM Ca ${ }^{2+}$ channels triggers CICR through the activated RyRs. These asymmetric $\mathrm{Ca}^{2+}$ signals accompanied by CICR are sufficient to trigger attraction (right).

Therefore, the NO-cGMP pathway can be regulated downstream of $\mathrm{Ca}^{2+}$ signals, in addition to its role upstream of CICR. The $\mathrm{Ca}^{2+}$-mediated nNOS regulation raises an intriguing possibility that neuronal electrical activity influences the turning behaviors of growth cones by changing the amount of cGMP via NO. An analogous mechanism has been reported in which cAMP acts downstream of $\mathrm{Ca}^{2+}$ for the regulation of axon guidance by electrical activity (Ming et al., 2001).

To test for the involvement of nNOS in axon guidance in vivo, we examined axon trajectories in nNOS knock-out mice at various developmental stages (embryonic days 10-13 and postnatal 0 ) by whole-mount and section immunohistochemistry (data not shown). Because our in vitro experiments were performed using DRG neuronal cultures supplemented with NGF, the corresponding subpopulation of DRG axons in vivo was labeled by an antibody against TrkA or calcitonin gene-related peptide, markers for the NGF-dependent nociceptive neurons. However, we found no detectable guidance errors of the DRG central and peripheral projections in nNOS knock-out mice. Although subtle axon guidance defects are often undetectable in mammals, our

observations suggest the presence of complex regulatory mechanisms of cyclic nucleotide signaling by various CAMs and extracellular matrices in vivo. Furthermore, the loss of nNOS could be compensated by supplying NO from different sources, e.g., by the action of eNOS or iNOS.

\section{Control of growth cone guidance by cyclic nucleotides}

Previous in vitro studies using Xenopus spinal neurons delineated two groups of diffusible guidance cues in terms of the modulatory effects of cyclic nucleotides on growth cone turning (Song and Poo, 1999). The turning responses to group I cues, such as netrin-1 and brain-derived neurotrophic factor (BDNF), depend on the cytosolic level of cAMP, whereas those induced by group II cues, such as Sema3A and neurotrophin-3, depend on the cGMP level instead. For both groups, elevating and lowering the levels of cyclic nucleotides favors growth cone attraction and repulsion, respectively. However, these grouping criteria have been revised by more recent work. For example, the growth cone turning response to netrin-1 depends not only on cAMP but also on cGMP (Nishiyama et al., 2003). This report provides evidence that the ratio of cAMP to cGMP sets the polarity of netrin-1-mediated guidance, with high ratios favoring attraction and low ratios causing repulsion. This notion is consistent with our conclusion that these two cyclic nucleotides control $\mathrm{Ca}^{2+}$-mediated axon guidance in a counteractive manner.

The role of cyclic nucleotides is complicated in axon guidance mediated by Sema3A that has been originally classified into group II. In chick DRG neurons, Sema3A-induced growth cone collapse is mediated by the cGMP-PKG pathway but abolished by activating the CAMP-PKA pathway, indicating the antagonizing effects by these two pathways (Dontchev and Letourneau, 2002; Chalasani et al., 2003). It has also been reported that, via activating the CAMP-PKA pathway, BDNF abolishes collapse of chick retinal growth cones induced by an exogenously applied NO donor (Gallo et al., 2002). These findings are well in agreement with our model. In Xenopus neurons, however, cyclic nucleotides regulate Sema3Ainduced growth cone behaviors in different ways. Asymmetric cGMP elevations across the growth cone that are not accompanied by $\mathrm{PKG}$ activation mediate Sema3A-induced repulsion by triggering $\mathrm{Ca}^{2+}$ influx through cyclic nucleotide-gated channels (Togashi et al., 2008). When cGMP has activated PKG in the growth cone, PKG causes membrane depolarization and thereby switches Sema3A-induced repulsion to attraction (Nishiyama et al., 2008). The differential roles of the cGMP-PKG pathway in Sema3A-mediated axon guidance could be explained by distinct expression profiles of ion chan- 
nels that are regulated downstream of PKG. In Xenopus spinal neurons, PKG causes depolarization by opening saxitoxinsensitive $\mathrm{Na}^{+}$channels, which stimulates $\mathrm{Ca}^{2+}$ influx through voltage-dependent $\mathrm{Ca}^{2+}$ channels, leading to growth cone attraction (Nishiyama et al., 2008). Conversely, cGMP inactivates RyRs via PKG in chick DRG neurons, allowing the generation of $\mathrm{Ca}^{2+}$ signals without CICR that trigger growth cone repulsion.

In conclusion, we have identified a novel regulatory mechanism of axon guidance that involves NO and cGMP acting at the level of cytosolic $\mathrm{Ca}^{2+}$ signals. This discovery will contribute to our understanding of intracellular signals that control the motile behaviors of growth cones during neuronal circuit development.

\section{References}

Calabrese V, Mancuso C, Calvani M, Rizzarelli E, Butterfield DA, Stella AM (2007) Nitric oxide in the central nervous system: neuroprotection versus neurotoxicity. Nat Rev Neurosci 8:766-775.

Castellani V, De Angelis E, Kenwrick S, Rougon G (2002) Cis and trans interactions of L1 with neuropilin-1 control axonal responses to semaphorin 3A. EMBO J 21:6348-6357.

Chalasani SH, Sabelko KA, Sunshine MJ, Littman DR, Raper JA (2003) A chemokine, SDF-1, reduces the effectiveness of multiple axonal repellents and is required for normal axon pathfinding. J Neurosci 23:1360-1371.

Chao DL, Ma L, Shen K (2009) Transient cell-cell interactions in neural circuit formation. Nat Rev Neurosci 10:262-271.

Dontchev VD, Letourneau PC (2002) Nerve growth factor and semaphorin $3 \mathrm{~A}$ signaling pathways interact in regulating sensory neuronal growth cone motility. J Neurosci 22:6659-6669.

Gallo G, Ernst AF, McLoon SC, Letourneau PC (2002) Transient PKA activity is required for initiation but not maintenance of BDNF-mediated protection from nitric oxide-induced growth-cone collapse. J Neurosci 22:5016-5023.

Gao Y, Nikulina E, Mellado W, Filbin MT (2003) Neurotrophins elevate cAMP to reach a threshold required to overcome inhibition by MAG through extracellular signal-regulated kinase-dependent inhibition of phosphodiesterase. J Neurosci 23:11770-11777.

Gomez TM, Robles E, Poo M, Spitzer NC (2001) Filopodial calcium transients promote substrate-dependent growth cone turning. Science 291:1983-1987.

Haase A, Bicker G (2003) Nitric oxide and cyclic nucleotides are regulators of neuronal migration in an insect embryo. Development 130:3977-3987.

Hajimohammadreza I, Raser KJ, Nath R, Nadimpalli R, Scott M, Wang KK (1997) Neuronal nitric oxide synthase and calmodulin-dependent protein kinase IIalpha undergo neurotoxin-induced proteolysis. J Neurochem 69:1006-1013.

Henley J, Poo MM (2004) Guiding neuronal growth cones using $\mathrm{Ca}^{2+}$ signals. Trends Cell Biol 14:320-330.

Henley JR, Huang KH, Wang D, Poo MM (2004) Calcium mediates bidirectional growth cone turning induced by myelin-associated glycoprotein. Neuron 44:909-916.

Hong K, Nishiyama M, Henley J, Tessier-Lavigne M, Poo M (2000) Calcium signalling in the guidance of nerve growth by netrin-1. Nature 403:93-98.

Höpker VH, Shewan D, Tessier-Lavigne M, Poo M, Holt C (1999) Growthcone attraction to netrin-1 is converted to repulsion by laminin-1. Nature 401:69-73.

Huang PL, Dawson TM, Bredt DS, Snyder SH, Fishman MC (1993) Targeted disruption of the neuronal nitric oxide synthase gene. Cell 75:1273-1286.

Jaffrey SR, Snyder SH (1995) Nitric oxide: a neural messenger. Annu Rev Cell Dev Biol 11:417-440.

Kamiguchi H, Yoshihara F (2001) The role of endocytic L1 trafficking in polarized adhesion and migration of nerve growth cones. J Neurosci 21:9194-9203.

Kojima H, Urano Y, Kikuchi K, Higuchi T, Hirata Y, Nagano T (1999) Fluorescent indicators for imaging nitric oxide production. Angew Chem Int Ed Engl 38:3209-3212.

Komeima K, Hayashi Y, Naito Y, Watanabe Y (2000) Inhibition of neuronal nitric-oxide synthase by calcium/calmodulin-dependent protein kinase
IIalpha through Ser847 phosphorylation in NG108-15 neuronal cells. J Biol Chem 275:28139-28143.

Kuwajima G, Futatsugi A, Niinobe M, Nakanishi S, Mikoshiba K (1992) Two types of ryanodine receptors in mouse brain: skeletal muscle type exclusively in Purkinje cells and cardiac muscle type in various neurons. Neuron 9:1133-1142.

Ming G, Henley J, Tessier-Lavigne M, Song H, Poo M (2001) Electrical activity modulates growth cone guidance by diffusible factors. Neuron 29:441-452.

Ming GL, Song HJ, Berninger B, Holt CE, Tessier-Lavigne M, Poo MM (1997) cAMP-dependent growth cone guidance by netrin-1. Neuron 19:1225-1235.

Nishimura K, Yoshihara F, Tojima T, Ooashi N, Yoon W, Mikoshiba K, Bennett V, Kamiguchi H (2003) L1-dependent neuritogenesis involves ankyrinB that mediates L1-CAM coupling with retrograde actin flow. J Cell Biol 163:1077-1088.

Nishiyama M, Hoshino A, Tsai L, Henley JR, Goshima Y, Tessier-Lavigne M, Poo MM, Hong K (2003) Cyclic AMP/GMP-dependent modulation of $\mathrm{Ca}^{2+}$ channels sets the polarity of nerve growth-cone turning. Nature 423:990-995.

Nishiyama M, von Schimmelmann MJ, Togashi K, Findley WM, Hong K (2008) Membrane potential shifts caused by diffusible guidance signals direct growth-cone turning. Nat Neurosci 11:762-771.

Ooashi N, Futatsugi A, Yoshihara F, Mikoshiba K, Kamiguchi H (2005) Cell adhesion molecules regulate $\mathrm{Ca}^{2+}$-mediated steering of growth cones via cyclic AMP and ryanodine receptor type 3. J Cell Biol 170:1159-1167.

Paves H, Saarma M (1997) Neurotrophins as in vitro growth cone guidance molecules for embryonic sensory neurons. Cell Tissue Res 290:285-297.

Qian Y, Chao DS, Santillano DR, Cornwell TL, Nairn AC, Greengard P, Lincoln TM, Bredt DS (1996) cGMP-dependent protein kinase in dorsal root ganglion: relationship with nitric oxide synthase and nociceptive neurons. J Neurosci 16:3130-3138.

Rialas CM, Nomizu M, Patterson M, Kleinman HK, Weston CA, Weeks BS (2000) Nitric oxide mediates laminin-induced neurite outgrowth in PC12 cells. Exp Cell Res 260:268-276.

Robles E, Huttenlocher A, Gomez TM (2003) Filopodial calcium transients regulate growth cone motility and guidance through local activation of calpain. Neuron 38:597-609.

Schmidt H, Werner M, Heppenstall PA, Henning M, Moré MI, Kühbandner S, Lewin GR, Hofmann F, Feil R, Rathjen FG (2002) cGMP-mediated signaling via cGKIalpha is required for the guidance and connectivity of sensory axons. J Cell Biol 159:489-498.

Song H, Ming G, He Z, Lehmann M, McKerracher L, Tessier-Lavigne M, Poo M (1998) Conversion of neuronal growth cone responses from repulsion to attraction by cyclic nucleotides. Science 281:1515-1518.

Song HJ, Poo MM (1999) Signal transduction underlying growth cone guidance by diffusible factors. Curr Opin Neurobiol 9:355-363.

Song HJ, Ming GL, Poo MM (1997) cAMP-induced switching in turning direction of nerve growth cones. Nature 388:275-279.

Stroissnigg H, Trancíková A, Descovich L, Fuhrmann J, Kutschera W, Kostan J, Meixner A, Nothias F, Propst F (2007) S-nitrosylation of microtubule-associated protein $1 \mathrm{~B}$ mediates nitric-oxide-induced axon retraction. Nat Cell Biol 9:1035-1045.

Su Z, Blazing MA, Fan D, George SE (1995) The calmodulin-nitric oxide synthase interaction. Critical role of the calmodulin latch domain in enzyme activation. J Biol Chem 270:29117-29122.

Suko J, Maurer-Fogy I, Plank B, Bertel O, Wyskovsky W, Hohenegger M, Hellmann G (1993) Phosphorylation of serine 2843 in ryanodine receptor-calcium release channel of skeletal muscle by cAMP-, cGMPand CaM-dependent protein kinase. Biochim Biophys Acta 1175:193-206.

Tessier-Lavigne M, Goodman CS (1996) The molecular biology of axon guidance. Science 274:1123-1133.

Thippeswamy T, McKay JS, Quinn J, Morris R (2005) Either nitric oxide or nerve growth factor is required for dorsal root ganglion neurons to survive during embryonic and neonatal development. Brain Res Dev Brain Res 154:153-164.

Togashi K, von Schimmelmann MJ, Nishiyama M, Lim CS, Yoshida N, Yun B, Molday RS, Goshima Y, Hong K (2008) Cyclic GMP-gated CNG channels function in Sema3A-induced growth cone repulsion. Neuron 58:694-707.

Tojima T, Akiyama H, Itofusa R, Li Y, Katayama H, Miyawaki A, Kamiguchi 
H (2007) Attractive axon guidance involves asymmetric membrane transport and exocytosis in the growth cone. Nat Neurosci 10:58-66.

Trimm KR, Rehder V (2004) Nitric oxide acts as a slow-down and search signal in developing neurites. Eur J Neurosci 19:809-818.

Wang GX, Poo MM (2005) Requirement of TRPC channels in netrin-1induced chemotropic turning of nerve growth cones. Nature 434:898-904.

Ward SM, Shuttleworth CW, Kenyon JL (1994) Dorsal root ganglion neurons of embryonic chicks contain nitric oxide synthase and respond to nitric oxide. Brain Res 648:249-258.

Wehrens XH, Lehnart SE, Reiken S, Vest JA, Wronska A, Marks AR (2006) Ryanodine receptor/calcium release channel PKA phosphorylation: a critical mediator of heart failure progression. Proc Natl Acad Sci U S A 103:511-518.

Welshhans K, Rehder V (2007) Nitric oxide regulates growth cone filopodial dynamics via ryanodine receptor-mediated calcium release. Eur J Neurosci 26:1537-1547.
Wen Z, Guirland C, Ming GL, Zheng JQ (2004) A CaMKII/calcineurin switch controls the direction of $\mathrm{Ca}^{2+}$-dependent growth cone guidance. Neuron 43:835-846.

Xiao B, Zhong G, Obayashi M, Yang D, Chen K, Walsh MP, Shimoni Y, Cheng H, Ter Keurs H, Chen SR (2006) Ser-2030, but not Ser-2808, is the major phosphorylation site in cardiac ryanodine receptors responding to protein kinase A activation upon beta-adrenergic stimulation in normal and failing hearts. Biochem J 396:7-16.

Zaccolo M, Movsesian MA (2007) cAMP and cGMP signaling cross-talk: role of phosphodiesterases and implications for cardiac pathophysiology. Circ Res 100:1569-1578.

Zheng JQ (2000) Turning of nerve growth cones induced by localized increases in intracellular calcium ions. Nature 403:89-93.

Zucchi R, Ronca-Testoni S (1997) The sarcoplasmic reticulum $\mathrm{Ca}^{2+}$ channel/ryanodine receptor: modulation by endogenous effectors, drugs and disease states. Pharmacol Rev 49:1-51. 